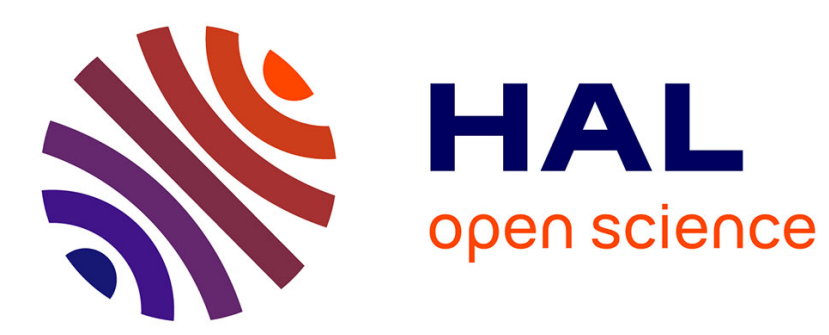

\title{
NEUTRONS AND 2 D ADSORBED \\ PHASESNEUTRON SCATTERING FROM 36Ar AND \\ 4He FILMS \\ K. Carneiro
}

\section{> To cite this version:}

K. Carneiro. NEUTRONS AND 2 D ADSORBED PHASESNEUTRON SCATTERING FROM 36Ar AND 4He FILMS. Journal de Physique Colloques, 1977, 38 (C4), pp.C4-1-C4-9. 10.1051/jphyscol:1977401 . jpa-00217117

\section{HAL Id: jpa-00217117 https://hal.science/jpa-00217117}

Submitted on 1 Jan 1977

HAL is a multi-disciplinary open access archive for the deposit and dissemination of scientific research documents, whether they are published or not. The documents may come from teaching and research institutions in France or abroad, or from public or private research centers.
L'archive ouverte pluridisciplinaire HAL, est destinée au dépôt et à la diffusion de documents scientifiques de niveau recherche, publiés ou non, émanant des établissements d'enseignement et de recherche français ou étrangers, des laboratoires publics ou privés. 


\title{
NEUTRON SCATTERING FROM ${ }^{36}$ Ar AND ${ }^{4}$ He FILMS
}

\author{
K. CARNEIRO \\ Physics Laboratory I, H. C. Ørsted Institute, \\ Universitetsparken 5, DK-2100 - Copenhagen $\varnothing$, Danemark.
}

\begin{abstract}
Résumé. - On indique, parmi les gaz couramment utilisés en adsorption, ceux qui sont les meilleurs candidats à des études de diffusion neutronique. Les différents types d'expériences possibles (diffraction, diffusion inélastique cohérente et incohérente), sont passés en revue et les informations pouvant être obtenues sont rappelées.

Les études de monocouche de ${ }^{36} \mathrm{Ar}$ adsorbé sur du grafoil montrent que l'argon forme des cristaux bidimensionnels délocalisés presque idéaux. Cette délocalisation donne cependant une déformation du réseau. Les études de ${ }^{4} \mathrm{He} /$ grafoil révèlent que des rotons analogues à ceux de volume existent dans des couches minces ayant seulement une épaisseur de quelques couches atomiques.

Abstract. - Scale factors for neutron diffraction and neutron inelastic scattering are presented for common adsorbates, and the feasibility of experiments is discussed together with the information gained by each type of experiment. Diffraction, coherent inelastic scattering, and incoherent scattering are treated separately. Studies of monolayers of ${ }^{36} \mathrm{Ar}$ adsorbed on grafoil show that argon forms almost ideal two-dimensional crystals, which are incommensurate with the substrate. The incommensurability gives, however, rise to a distortion of the lattice. Studies of ${ }^{4} \mathrm{He}$ on grafoil reveal that bulklike rotons exist in films of only a few atomic layers thickness.
\end{abstract}

1. Introduction. - The technique of neutron scattering is well established as a unique tool to investigate the details of the microscopic structure and dynamics of bulk matter, and a desire to apply this fascinating experimental technique to physisorbed phases is quite natural. But on the other hand since neutron scattering, compared to other spectroscopic methods such as nuclear magnetic resonance and scattering by $\mathrm{X}$-rays and light, is notable for its very low yield of intensity, it seems at first sight a headless task to start an investigation with neutrons on for instance a mono-molecular layer film, where typically $99 \%$ of the material that scatters neutrons is the substrate, which we are not particularly interested in.

The fact that an appreciable part of this conference is devoted to the discussion of neutron scattering results shows that the substrate problem has been solved to the advantage of the physisorbed films. A major breakthrough was the occurrence of grafoil which, besides providing a high quality surface increasing the homogeneity of the film, in the case of neutron scattering gives an unusual possibility of discriminating between the neutron scattering from the adsorbed gas and that from the underlying substrate. This latter advantage is closely related to the preferred orientation of the graphite flakes in the grafoil, so that one can perform the scattering experiment either in the in plane geometry, probing primarily the correlations (static or dynamic) within the plane of the film or (by turning the adsorption cell $90^{\circ}$ ) in the out of plane geometry, probing primarily correlations perpendicular to this plane.

In this paper I shall review the results obtained from films of $\mathrm{Ar}$ and ${ }^{4} \mathrm{He}$, adsorbed on grafoil. The Ar experiments [1, 2], performed at Brookhaven National Laboratory, constitute a fairly complete study, in both the above mentioned scattering geometries, of a physisorbed monolayer and relate both to the intrinsic physics of two-dimensional matter as well as to the interactions between the film and the substrate. Since we are still, even in the presence of this interaction, primarily interested in the film, I shall refer to this interaction as the perturbation of the properties of the monolayer by the substrate. The ${ }^{4} \mathrm{He}$ experiments [3], also carried out at Brookhaven, showed that although the neutron scattering cross section of this isotope is extremely small, one can still determine the structure of an adsorbed monolayer. But the primary objective of this study was to investigate in thin films (few atomic layers) the characteristics of the roton, the microscopic excitation which in bulk ${ }^{4} \mathrm{He}$ has fascinated scientists for decades.

Before going into detail about what physics has been learned from these two studies, it seems appropriate in the spirit of this conference to use the $\mathrm{Ar}$ and $\mathrm{He}$ experiments in the context of neutron scattering methodology. They are in certain ways 
TABLE I.

Parameters of importance for the neutron scattering properties of some mono- and diatomic species, used in physisorption studies. As explained in the text, $\sigma_{0}$ and $\sigma_{1}$ are the scale factor for Bragg-scattering and phonon scattering respectively.

$\begin{array}{cc}\text { Species } & \begin{array}{c}b_{\mathrm{COH}}{ }^{+} \\ \left(10^{-12} \mathrm{~cm} / \text { nucleus }\right.\end{array} \\ & \\ { }^{3} \mathrm{He}{ }^{*} & 0.61 \\ { }^{4} \mathrm{He} & 0.30 \\ \mathrm{Ne} & 0.46 \\ { }^{36} \mathrm{Ar} & 2.43 \\ \mathrm{Kr} & 0.74 \\ \mathrm{Xe} & 0.48 \\ \mathrm{H}_{2} & 0.374 \\ \mathrm{D}_{2} & 0.667 \\ \mathrm{~N}_{2} & 0.940 \\ \mathrm{O}_{2} & 0.580 \\ \mathrm{Cl}_{2} & 0.958 \\ \mathrm{C}^{* *} & \end{array}$

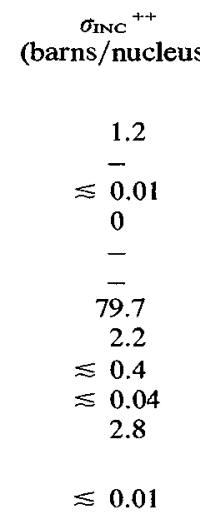

$\sigma_{\text {ABS }}^{++}(\lambda=1.08$
(barns $/$ nucleus $)$
3200
$\sim 0$
0.2
0.4
18
18
0.19
0.0005
1.1
0.0001
19.5
0.003

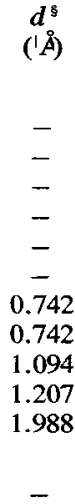

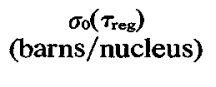

$\begin{array}{rl}4.9 & 4.74 \\ 1.1 & 0.67 \\ 2.7 & 0.14 \\ 74.2 & 1.64 \\ 6.9 & 0.06 \\ 2.9 & 0.02 \\ 6.1 & 1.85 \\ 19.5 & 2.95 \\ 32.8 & 0.82 \\ 11.6 & 0.27 \\ 15.5 & 0.17 \\ & 0.04 \text { (ip) } \\ 5.6 & 0.19 \text { (op) }\end{array}$

$+b_{\mathrm{COH}}$, the coherent scattering amplitudes, are taken from the compilation by C.G. Shull (private communication -1972 ).

${ }^{++} \sigma_{\mathrm{INC}}$ and $\sigma_{\mathrm{ABS}}$, the cross sections for incoherent scattering and for true absorption, are taken from Neutron Diffraction (G.E. Bacon, Oxford at the Clarendon), 1962, p. 31 and p. 61

${ }^{8} \boldsymbol{d}$, molecular bond length are taken from Molecular Spectra and Molecular Structure, I Spectra of diatomic molecules (G. Herzberg, D. Van Nostrand and Co., New York), 1950.

* The data for ${ }^{3} \mathrm{He}$ is taken from Ref. 9.

$* *$ ip $=$ in plane, op = out of plane (See text).

prototype experiments which provide us with enough experience to predict the feasibility of future projects.

2. Neutron scattering methodology. - Table I shows the relevant neutron parameters for some of the mono- and diatomic molecules of interest for physisorption studies. These are the coherent scattering amplitude per bound nucleus, $b_{\mathrm{COH}}$, the spin incoherent scattering cross section $\sigma_{\mathrm{INC}}$, and the true absorption cross section $\sigma_{\mathrm{ABS}}$. In order to see how these parameters influence the scattered intensities we have to distinguish between the three cases: Diffraction, coherent inelastic scattering, and incoherent scattering.

2.1. DifFraCtion STUDIES. - Let us consider an infinite two-dimensional (2-D) crystal with one molecule per unit cell, for which the elastic neutron scattering cross-section per molecule is the following :

$$
\frac{\mathrm{d} \sigma}{\mathrm{d} \Omega}=\frac{\sigma_{0}(Q)}{4 \pi} \mathrm{e}^{-2 W(\boldsymbol{O})} \sum_{\tau} \delta(\mathbf{Q}-\tau)
$$

where $\mathbf{Q}$ is the wavevector transferred to the crystal by the neutrons, $\tau$ is a reciprocal lattice vector, and $\mathrm{e}^{-2 w(0)}$ is the Debye-Waller factor, with $W(\mathbf{Q})=\frac{1}{2}\left\langle(\mathbf{Q} \cdot \mathbf{u})^{2}\right\rangle$ where $\mathbf{u}$ is the vector displacement of an atom from its mean position, and $\langle>$ denotes ensemble average. Since $\tau$ is within the plane of the crystal, (1) applies for the in plane scattering geometry. The scale factor for the diffracted (or elastically scattered) intensities are :

$\sigma_{0}(Q)=\left\{\begin{array}{l}4 \pi b_{\mathrm{COH}}^{2}, \text { for monoatomic gases } \\ 4 \pi\left[2 b_{\mathrm{COH}} j_{0}(Q d / 2)\right]^{2},\end{array}\right.$

for diatomic gases

where $j_{0}(x)=(\sin x) / x$ is the zeroth order spherical Bessel function, and $d$ is the molecular bond length, also listed in table $I$. Thus for small Q's, where $j_{0}$ is close to unity a substantial enhancement of the intensities in the diffraction lines is obtained for the diatomic gases, at the expense of cancellation of intensity at the zero's of $j_{0} . j_{0}(Q d / 2)$ plays the role of a molecular form factor and applies strictly speaking only to the case where the orientations of molecules are uncorrelated [4], but numerically it differs little from what one obtains from angularly correlated molecules.

Using the reciprocal lattice vector $\tau_{\text {reg }}=1.703 \AA^{-1}$ for the well known registered lattice, which is formed by one third of the graphite substrate hexagons, table I shows $\sigma_{0}(Q)$ for $Q=\tau_{\text {reg }}$. It is not astonishing that the trial experiment was chosen to be $\mathrm{N}_{2}$ adsorbed on grafoil [5], and with this successfully done, a study of the separated isotope ${ }^{36} \mathrm{Ar}$ was initiated. Diffraction profiles from monolayers of ${ }^{36} \mathrm{Ar}$ are shown in figure 1 , using neutron energies of $13 \mathrm{meV}$ (i.e., wavelength $\lambda=2.5 \AA$ ). The observed sawtooth lineshape, 


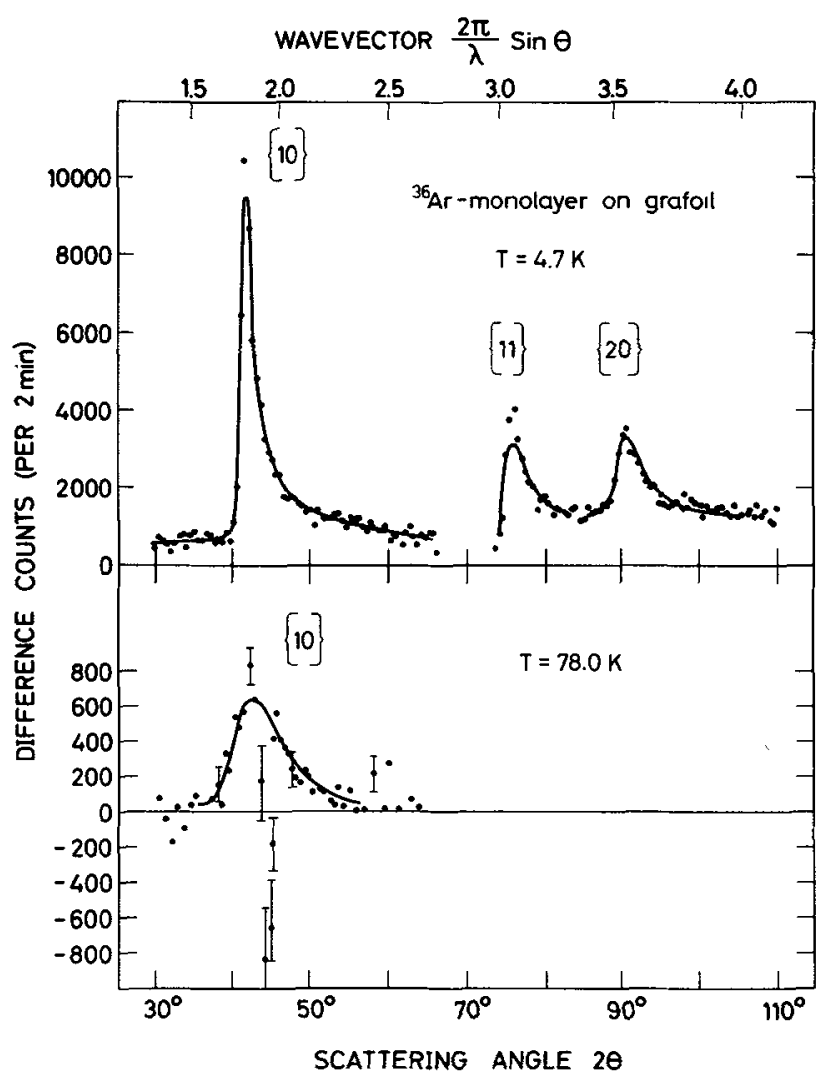

FIG. 1. - Neutron elastic scattering from monolayers of ${ }^{36} \mathrm{Ar}$ adsorbed on grafoil, at the two temperatures 4.7 and $78.0 \mathrm{Kelvin}$.

characteristic of diffraction from many (2-D) crystals, of which the planes are not parallel, was first explained by Warren [6] for an isotropic distribution of planes, and has later been extended to the case of preferred orientation $[2,5]$, e.g., when the (2-D) crystals are adsorbed on grafoil. Based on the Warren model we can analyze the data in the upper part of figure 1 as follows :

1) The position of the diffraction line is determined by the unit cell parameters of the film. For argon at $T=4.7 \mathrm{~K}$, we get $\tau_{10}=1.89 \AA^{-1}$, corresponding to a nearest neighbour distance of the triangular lattice $a_{\mathrm{nn}}=3.86 \AA$.

2) The relative intensities of the diffraction lines is determined by the Debye-Waller factor, which in the case of argon at $T=4.7 \mathrm{~K}$ gives $2 W(Q) \approx 0.05 \AA^{2} \cdot Q^{2}$.

3) The slope of the leading edge of the diffraction line is determined by the diameter, $L$, of the (2-D) crystal after correction for instrumental resolution. When finite sizes of diffracting crystals are considered, (1) gets modified and in our example we get $L \approx 110 \AA$. The trailing edge of the line is determined jointly by the Debye-Waller factor and the orientational distribution functions of the film planes, and does not yield direct information of interest for the study of the (2-D) crystal.
Hence, although the diffraction lines of the upper part of figure 1 are not simple $\delta$-functions as prescribed by (1) we are able to extract the unit cell parameters, the Debye-Waller factor, and the typical diameters of the (2-D) crystals adsorbed on grafoil. However, with the experience gained with the strongest neutron scatterers $\mathrm{N}_{2}$ and ${ }^{36} \mathrm{Ar}$, it proved possible to investigate the structure of a large variety of adsorbed gases, and work is now going on to map out the phase diagrams of $\mathrm{H}_{2}, \mathrm{D}_{2}{ }^{3} \mathrm{He}$ and $\mathrm{O}_{2}$ [7], as well as $\mathrm{Kr}$ [8]. In figure 2 we show the diffraction line from a monolayer of ${ }^{4} \mathrm{He}[3]$, the weakest scatterer according to table $I$, indicating that the structure of any molecular substance can be investigated. Allowing some differences in the Debye-Waller factors, the observed intensities of ${ }^{36} \mathrm{Ar}$ and ${ }^{4} \mathrm{He}$ scale approximately as their $\sigma_{0}$. It is remarkable that the enormous $(n, \gamma)$ absorption cross section of ${ }^{3} \mathrm{He}$, which in bulk causes serious difficulties and does in fact prevent direct structural studies [9], allows diffraction studies of (2-D) phases. Here the substrate helps! It spreads out the gas, giving a tolerable mean free path for the neutrons.

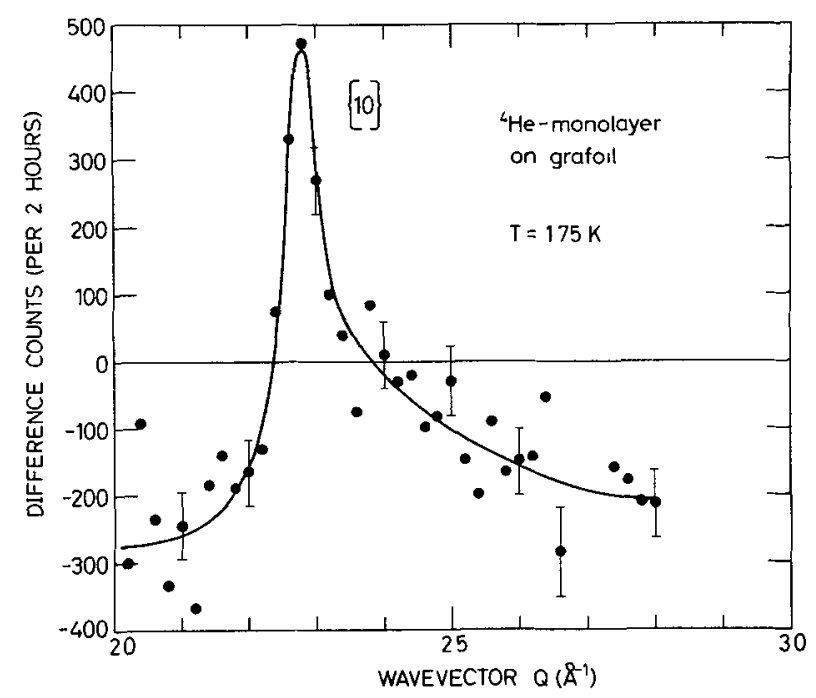

FIG. 2. - Neutron elastic scattering from monolayers of ${ }^{4} \mathrm{He}$, adsorbed on grafoil, at the temperature 1.75 Kelvin.

The lower part of figure 1 shows diffraction from monolayers of ${ }^{36} \mathrm{Ar}$ at high temperatures. Besides showing a change of the parameters $\tau, W(Q)$, and $L$ compared to their low temperature values, a significant negative scattering is observed. This effect is related to the distance, $z$, between the plane of the film and the adsorption surface, since the difference in scattering amplitudes and in repeat distance changes the shape of the diffraction in the $(00 l)$ direction of the substrate. This out of plane effect, which shows up in the in plane scattering because of misoriented graphite flakes, was in the case of argon [2] used to estimate that $z=4.1 \AA$. 
Experimentally, there are various ways to discriminate against the graphite scattering. Since the scattering according to (1) is strictly elastic, it is profitable to use an energy analyzer in the outgoing beam, set at the same energy as the monochromator. Also by keeping the neutron energy below the Bragg cut-off for in plane diffraction in the substrate, the background intensity can be markedly improved.

2.2 COHERENT INELASTIC SCATTERING. Turning now to the dynamical properties of the ideal (2-D) crystal considered above, the double differential cross section for scattering of neutrons from the initial energy $E_{0}=\hbar^{2} k_{0}^{2} / 2 m,\left(k_{0}\right.$ is the wavevector) to the lower energy $E=\hbar^{2} k^{2} / 2 m$ becomes :

$$
\begin{aligned}
\frac{\mathrm{d}^{2} \sigma_{\text {COH }}}{\mathrm{d} \Omega \mathrm{d} \omega} & =\frac{k}{k_{0}} \frac{\sigma_{0}}{4 \pi} \frac{n(\omega)+1}{M \omega} \mathrm{e}^{-2 \omega(\mathbf{Q})} \sum_{\mathbf{t} \mathbf{q}} \sum_{j=1}^{2} \times \\
& \times\left(\mathbf{Q} \cdot \sigma_{i}\right)^{2} \delta\left(\omega-\omega_{j}(\mathbf{q})\right) \times \delta(\mathbf{Q}-\tau \pm \mathbf{q})
\end{aligned}
$$

where $M$ is the molecular mass $n(\omega)=\left[\exp \left(\hbar \omega / k_{\mathrm{B}} T\right)-1\right]^{-1}$ is the Bose-Einstein population factor, $\hbar \omega=E_{0}-E$ and $\mathbf{Q}=\mathbf{k}_{0}-\mathbf{k} \cdot \omega_{j}$ is the phonon frequency of the $j$ 'th normal mode of wavevector $q$ and polarization $\sigma_{j} \cdot j=1$ and 2 denotes longitudinal $\left(\boldsymbol{\sigma}_{1} \| \mathbf{q}\right)$ and transverse $\left(\boldsymbol{\sigma}_{2} \perp \mathbf{q}\right)$ respectively. From (2) we see that a scale factor for inelastic scattering may be defined as :

$$
\sigma_{1}(Q)=\sigma_{0} \frac{\hbar Q^{2}}{M \omega_{1}}
$$

where $\omega_{1}$ is a characteristic frequency. Using the 3-D Debye-frequencies and again setting $Q=\tau_{\text {reg }}$, I show in table I $\sigma_{1}\left(\tau_{\text {reg }}\right)$.

The inelastic scale factors, combined with our gained experience, indicate that the number of candidates for inelastic scattering from adsorbed phases is rather limited, since in the most favorable cases $\mathrm{Ar}, \mathrm{H}_{2}$ and $\mathrm{D}_{2}$, which have been studied, long counting times are needed, even to give marginal intensities. The helium isotopes, however, form an exception from the rule. In liquid ${ }^{4} \mathrm{He}$ the cross-section (3) must be modified by setting $\tau=\mathbf{0}$ and $\mathbf{q}=\mathbf{Q}$, but the very special excitation spectrum causes the scattered intensity to concentrate at essentially the roton minimum, whereas a normal Debye-spectrum yields intensities at many wavevectors. Consequently the roton could be rather well studied as shown in figure 3 . On the other hand, the absorption of ${ }^{3} \mathrm{He}$ seems at the moment to prevent inelastic studies from this isotope. In the other cases of table $\mathrm{I}$, absorption does however not seem of importance.

To go from the ideal expression (3) to the actual inelastic intensities from films on grafoil requires a procedure similar to that employed in the diffraction case.

Without going into detail, let me mention the more

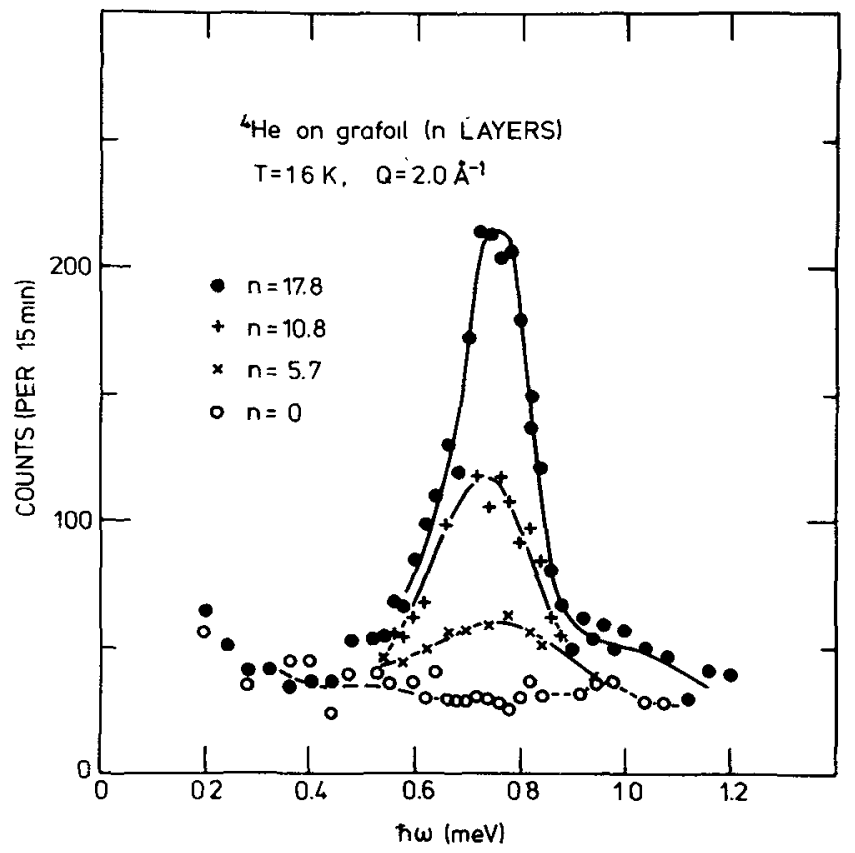

FIG. 3. - Neutron inelastic scattering from grafoil (open circles) and from grafoil with adsorbed ${ }^{4} \mathrm{He}$. The momentum transfer $Q$ is paralle1 to the average film plane in the cell (In plane geometry).

general observation from monolayers of argon, obtained in the in plane geometry :

1) At low temperatures $T=4.7 \mathrm{~K}$ the intensities are in accordance with (3) with $\omega_{j}$ taken to be the phonon frequencies of a (2-D) crystal, in quantitative agreement with nearest-neighbour forces according to Lennard-Jones interaction parameters, as determined from gas phase measurements. We found that the sound velocities $\left(\mathrm{d} \omega_{j} / \mathrm{d} q\right.$ at small $\left.q^{\prime} \mathrm{s}\right)$ in the (2-D) argon crystal was $v_{1}=1650 \mathrm{~m} / \mathrm{s}(\mathrm{LA})$ and $v_{2}=900 \mathrm{~m} / \mathrm{s}(\mathrm{TA})$. As for the Debye-Waller factor, the intensities indicate that $2 W(Q)=0.27 \AA^{2} Q^{2}$.

2) At intermediate temperatures the neutron spectrum broadens and there is an indication that the transverse phonon branch softens ( $v_{2}$ goes down). At higher temperatures $(T \geqslant 40 \mathrm{~K})$ the spectrum looks quasi-elastic. It should, however, be noted that the quasi-elastic neutron scattering here is a result of phonon damping and that it is not in a simple way related to particle mobility. Formally the effect may be taken into account in (3) by changing the $\delta\left(\omega-\omega_{j}\right)$ term into a lorentzian with a width $\Gamma_{j}$ centered around $\omega_{j}$.

Some results of neutron inelastic scattering from the absorption cell, obtained in the out of plane geometry is shown in figure 4, with and without a monolayer of argon in the cell. The difference intensities shown in figure 5 indicate a resonance at $\hbar \omega_{\perp}=5.4 \mathrm{meV}$ independent of the wavevector $\mathbf{Q}$. This shows that the argon atoms move essentially uncoupled perpendicular to the adsorption surface, 


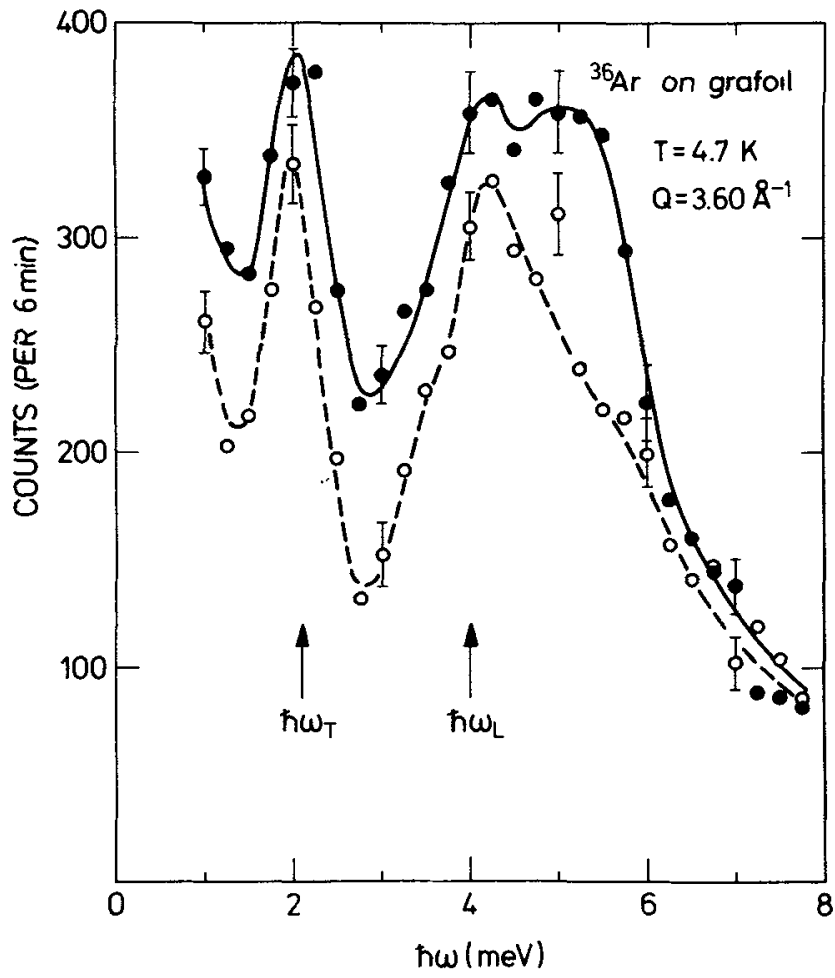

FIG. 4. - Neutron inelastic scattering from grafoil (open circles), and from grafoil with adsorbed ${ }^{36} \mathrm{Ar}$-monolayers (closed circles). The momentum transfer $Q$ is oriented perpendicular to the average film plane in the cell (out of plane geometry). $\omega_{\mathrm{T}}$ and $\omega_{\mathrm{L}}$ are calculated as described in the text.

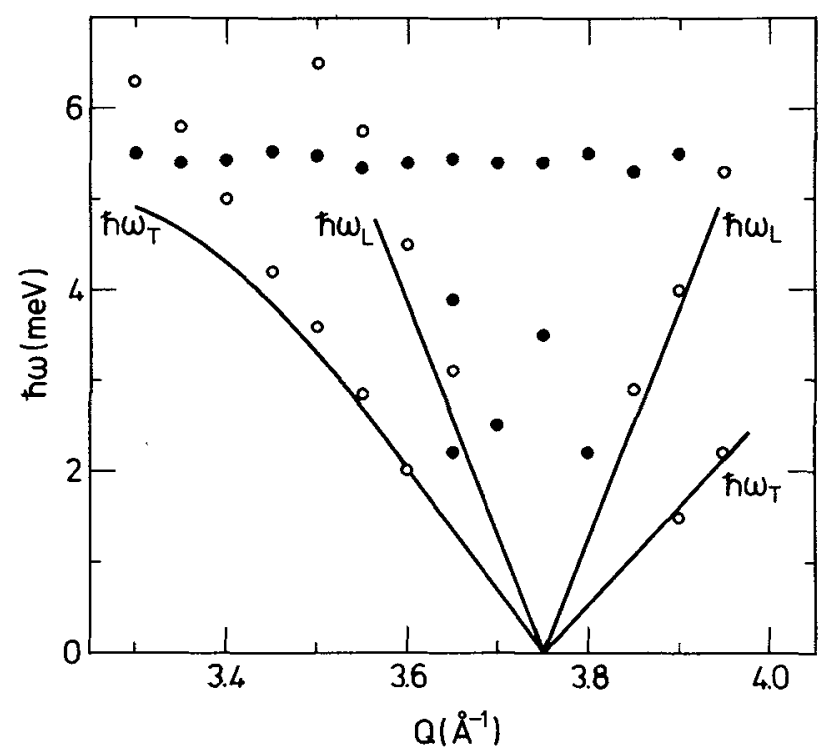

FIG. 5. - Results of the out of plane scattering from monolayers of ${ }^{36} \mathrm{Ar}$ on grafoil. The open circles show the graphite phonons and the solid lines are calculated as explained in the text. The solid circles show the characteristic out of plane Ar resonance at $\hbar \omega=5.4 \mathrm{meV}$ together with some accidental in plane phonons at lower frequencies. so that analogous to (3) we get for the out of plane scattering :

$\begin{aligned} \frac{\mathrm{d}^{2} \sigma_{\mathrm{COH}}}{\mathrm{d} \Omega}=\frac{k}{k_{0}} \frac{\sigma_{0}}{4 \pi} & \frac{n(\omega)+1}{M \omega} \times \\ & \times \mathrm{e}^{-2 \omega(\boldsymbol{O})}\left(\mathbf{Q} \cdot \sigma_{\perp}\right)^{2} \delta\left(\omega-\omega_{\perp}\right) .\end{aligned}$

At some wavevectors there are extra maxima in the scattering from the argon layer in the out of plane geometry. These are associated with in-plane scattering according to (3) which accidentally shows up in the out of plane geometry because of the misorientation of the grafoil.

The inelastic experiments [7] on the commensurate phases of $\mathrm{H}_{2}$ and $\mathrm{D}_{2}$ in the in plane geometry show a pattern similar to the out of plane spectrum of argon, indicating that the molecules perform uncoupled (or Einstein-like) oscillations of frequency $\omega_{H_{1}}$ within the film plane. This must originate from the variations of the molecule-graphite potential over the surface, which here in contrast to argon dominate molecular interactions, so that in (3) $\omega_{j}(Q)=\omega_{\|}$.

2.3 INCOHERENT SCATTERING. - Whereas neutrons couple to the collective correlations of matter via the coherent cross-section $\sigma_{\mathrm{COH}}=4 \pi b_{\mathrm{COH}}^{2}$, the spin incoherent scattering cross-section $\sigma_{\text {INC }}$ relates to single particle correlations. Evidently, from table I, protons are excellent candidates for such studies. Neglecting molecular form factors the double differential incoherent cross-section for the (2-D) crystal considered above is :

$$
\begin{aligned}
& \frac{\mathrm{d}^{2} \sigma_{\mathrm{INC}}}{\mathrm{d} \Omega \mathrm{d} \omega}=\frac{\sigma_{\mathrm{INC}}}{4 \pi} \mathrm{e}^{-2 W(\mathrm{Q})} \delta(\omega)+ \\
& \quad+\frac{k}{k_{0}} \frac{\sigma_{\mathrm{INC}}}{4 \pi} \frac{n(\omega)+1}{M \omega} \mathrm{e}^{-2 W(Q)} \sum_{j=1}^{2}\left(\mathrm{Q} \cdot \sigma_{j}\right)^{2} Z_{j}(\omega)
\end{aligned}
$$

where the first term is elastic, and the latter is analogous to (4) but with the density of state $z(\omega)$ instead of the specific frequencies. Note that in the case of uncoupled Einstein oscillators of frequency $\omega_{\mathrm{E}}, Z(\omega)=\delta\left(\omega-\omega_{\mathrm{E}}\right)$ and the inelastic incoherent scattering is identical to the coherent, which is only natural, since the difference between the two types of scattering originates precisely from the coupling between oscillators.

If on the other hand the scattering molecules do not form a lattice but are more or less freely moving, the incoherent scattering reflects the particle mobility. The elastic part of (6) signifying the static crystal, broadens into quasi-elastic scattering and in the case of simple diffusion according to Fick's law, with diffusion constant $D$, one gets :

$$
\frac{\mathrm{d}^{2} \sigma}{\mathrm{d} \Omega \mathrm{d} \omega}=\frac{\sigma_{\mathrm{INC}}}{4 \pi} \frac{1}{\pi} \frac{D Q^{2}}{\omega^{2}+\left(D Q^{2}\right)^{2}} \text {. }
$$

This is, however, only one of many models, which for instance does not take into account that genuine 
inelastic scattering corresponding to the second part in (6) may also be important.

Incoherent scattering has been widely used to study mobilities in bulk liquids and gases of molecules containing hydrogen, and the application to films is a subject of a separate paper [10]. $\mathrm{H}_{2}$ itself, where the molecular symmetry requires that only ortho-hydrogen scatterers incoherently at low temperatures, has also been studied [11].

3. Argon monolayers. - As mentioned above, argon adsorbed on grafoil forms a dense triangular lattice, with a low temperature lattice constant $a_{\mathrm{nn}}=3.86 \AA^{2}$. This appears to be independent of the filling indicating coexistence between the solid and a vapour phase. As the temperature is raised, the lattice expands at the expense of the necessarily available vapour, with a rather large linear thermal expansion coefficient of $\alpha=2 \times 10^{-3} \mathrm{~K}^{-1}$. The bulk value is $\alpha=0.4 \times 10^{-3} \mathrm{~K}^{-1}$. Another obvious difference between the monolayer and the bulk is the gradual decrease of the crystal diameter $L$ as the temperature is raised. At low temperatures $L=110 \AA$ and this value appears to be limited by the substrate; but at $T=70 \mathrm{~K}, L$ is about $20 \AA$, so small that one should probably identify $L$ as a correlation length and not a well-defined crystal size. The change in $L$ is, however, gradual with no abrupt sign of a melting transition. The diffraction intensity, governed by the Debye-Waller factor in (1) gives some indication of a melting transition at $T=55 \mathrm{~K}$. Also the intensity variation is gradual so that at $T=50 \mathrm{~K}$ one gets $2 W \approx-\left(0.35 \AA^{3}\right) Q^{2}$, compared to $2 W=-\left(0.05 \AA^{2}\right) Q^{2}$ at $T=5 \mathrm{~K}$; but above $T=60 \mathrm{~K}$ there is little change in the intensity. The high temperature phase of the monolayer, when interpreted via this Warren formalism, has a nearestneighbour distance of $4.26 \AA$, which matches the commensurate phase. This is similar to what was found for $N_{2}$ [5], indicating the existence of a registered liquid.

It is interesting to relate our observations to the theoretical picture of $(2-D)$ matter. Probably the most intriguing question is whether long range order exists in two dimensions, since positional order leads to a divergence of $\left\langle u^{2}\right\rangle$ whereas angular order does not. Our low temperature results show that within the limits set by our substrate, the size of $L$ can be large enough to give well defined diffraction peaks and to sustain well defined lattice excitations. Since it is incommensurate with the substrate, the argon monolayer is to some extent ideal $(2-D)$ like, with lattice constant and phonon spectrum in accordance with nearest-neighbour van der Waal's forces between argon atoms.

Viewed as ideal $(2-D)$ argon crystals we can interprete the thermal behaviour of the monolayers as follows. Using the Debye-Waller factor as order parameter we conclude that the crystals melt at
$T \approx 55 \mathrm{~K}$, where the transition is at least of second order. If we use the relation between $\left\langle\boldsymbol{u}^{2}\right\rangle$ and $W(Q)$, this is in perfect agreement with a computer simulation [12] of the melting of a $(2-D)$ Lennard Jones crystal, which used the divergence of $\left\langle\boldsymbol{u}^{2}\right\rangle$ to indicate the onset of melting. Considering the microscopic mechanism of the melting, our results lend support to the dislocation theory. Spontaneous formations of dislocations (or pairs of dislocations) can from an energetical point of view take place at characteristic melting temperatures, and they have been observed in computer simulation studies [13]. Dislocations will break the ideal symmetry of the lattice in a way which may appear as a decrease in our crystal diameter $L$, and they may couple to the transverse modes, thereby decreasing their frequency. Both effects are in accordance with our observations.

However, the picture of an ideal $(2-D)$ crystal becomes inconsistent when the Debye-Waller factor is analyzed in more detail. From the measured $(2-D)$ phonon spectrum and out of plane motion, we calculate the dynamical contribution to $\left\langle u^{2}\right\rangle$ to be $0.06 \AA^{2}$ at $T=0 \mathrm{~K}$, yielding $2 W=\left(0.02 \AA^{2}\right) Q^{2}$, significantly less than observed, both by diffraction and from the phonon intensities. Further, the $\{11\}$ diffraction intensity is

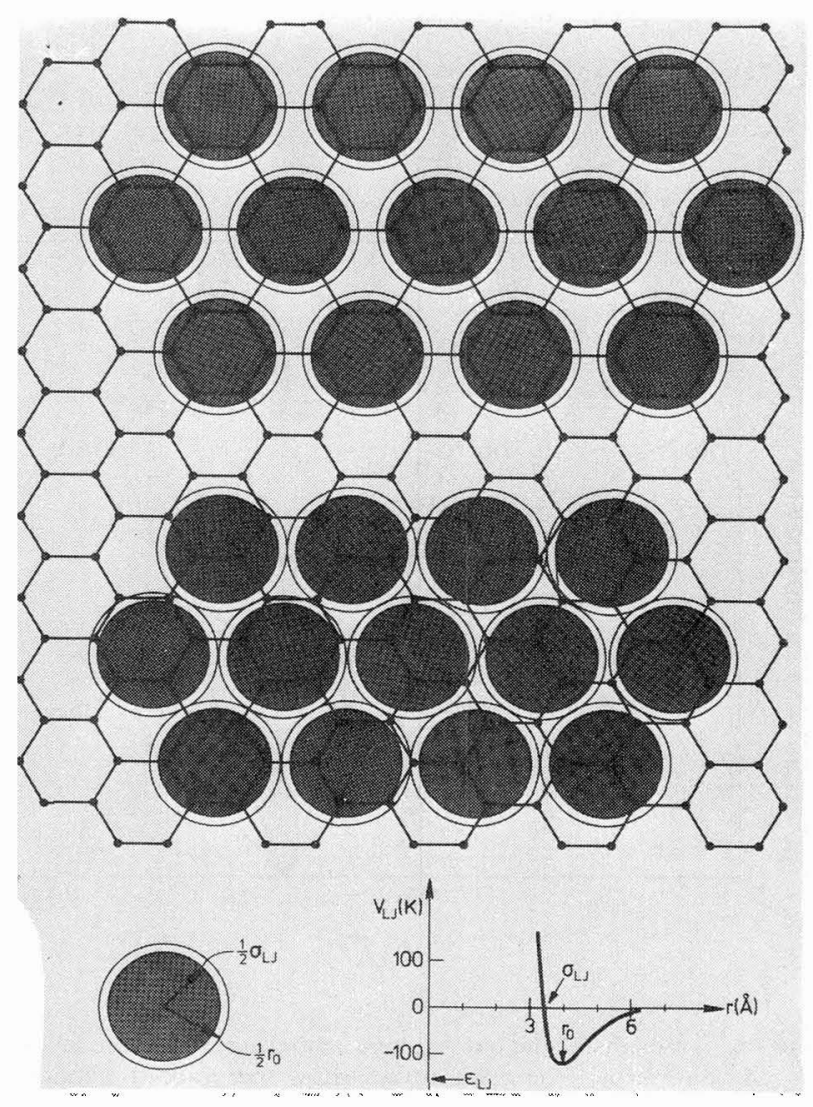

FIG. 6. - Arrangements of molecules on graphite. Upper part shows the commensurate phase, and lower part the dense phase. The potential parameters are relevant for argon. 
lower than the $\{20\}$, hinting that the Debye-Waller factor is anisotropic. To understand these effects, we must take the substrate into account. In figure 6 is shown the graphite adsorption surface covered with monolayers of argon both in the hypothetical, registered and the real, dense phase. In this context the major difference between the two cases is that in the dense case the substrate strains the monolayer, resulting in a static distortion contributing to $\left\langle\boldsymbol{u}^{2}\right\rangle$. Further if the layer is oriented in a well defined way with respect to the substrate hexagons, the strain will be anisotropic. The experimental indication from diffraction that the Debye-Waller factor is anisotropic support the picture of angular lock-in, which both recent calculations and more compelling experimental evidence show to occur [14]. From the inelastic scattering we get an appreciably larger $W(Q)$ than from diffraction. This may be due to that phonons propagate in all directions yielding an angular mean, whereas our diffraction is limited to a few high symmetry directions, but it may also come from the fact that the static distortion influences the phonon and diffraction intensities differently. To investigate the evidence for a direct coupling from the motion of the substrate to the monolayers, let us return to figure 5 . Both the scale factors $\sigma_{1}$ and the direct way in which graphite phonons polarized along the c-axis (i.e., out of plane) can perturb the film, we expect such a coupling to show up where the graphite out of plane phonons cross the argon oscillatory spectrum below $\hbar \omega=6 \mathrm{meV}$. Based on the known phonon spectrum of graphite, and taking the scattering geometry as well as the orientational distribution into account, I have computed the expected dispersion curves for grafoil for the transverse $\left(\omega_{T}\right)$ and longitudinal $\left(\omega_{L}\right)$ acoustic frequencies, in nice agreement with the measurements from the empty cell. But the monolayer does not respond at these frequencies, indicating the lack of direct dynamical coupling between the substrate and the incommensurate, physisorbed monolayer.

In conclusion, our neutron scattering experiments show that monolayers of argon on grafoil, to a certain degree behave as ideal $(2-D)$ crystals, on which the static strain imposed by the substrate has only a minor effect.

4. Thin ${ }^{4} \mathrm{He}$ films. - ${ }^{4} \mathrm{He}$ on grafoil has been studied extensively by macroscopic methods, and peculiar effects have been reported from films containing several atomic layers. It seems natural to relate these observations to the properties of a superfluid in a restricted geometry, in which either the roton energy $\hbar \Delta$ is changed from its bulk value, or new excitations at the free surface of the film, ripplons occur. Some of the inelastic neutron scattering results are shown in figure 3, and in ref. [3] it was concluded that down to 5.7 atomic layers, out of which at least two are solid, neither evidence of a change in $\Delta$ is seen nor any sign of a lower lying excitation is visible. In all scans of figure 3 , the bulk roton energy appears at $\hbar \omega=0.74 \mathrm{meV}$.

It is well known that $\Delta$ changes rather dramatically with density in bulk helium, so the well defined roton energy indicates that the excitations occur in a part of the film of well defined density. This is astonishing since one expects a drastic density gradient across the film, starting at the substrate with a density corresponding to several hundred atmospheres and ending at the surface in equilibrium with gas. Secondly, third sound measurements show that the superfluid density $\rho_{\text {s }}$ does change with coverage [16], and since we cannot from our results invoke the existence of ripplons as being responsible for the change in $\rho_{\mathrm{s}}, \Delta$ should change to account for the change in third sound ; and we observe no change. To quantify this dilemma I compare in figure 7 the observed roton energies to calculation, based on the third sound measurements and a simple model.

In bulk ${ }^{4} \mathrm{He}$ there is a semi-empirical relation [17] between the superfluid fraction $\rho_{\mathrm{s}} / \rho$ and the temperature relative to the $\lambda$-point :

$$
\rho_{\mathrm{s}} / \rho=\Phi_{1}\left(T_{\lambda}-T_{3 \mathrm{D}}\right)
$$

which, knowing the actual superfluid fraction of the film may be used to calculate an effective temperature $T_{3 \mathrm{D}}$. This may in turn be used to calculate $\Delta$ from the empirical relation between $T_{3 \mathrm{D}}$ and $\Delta$ :

$$
\Delta=\Phi_{2}\left(\rho, T_{3 \mathrm{D}}\right)
$$

where this relation is known from neutron scattering [18]. Via (8) and (9), the measured $\rho_{\mathrm{s}}$ is

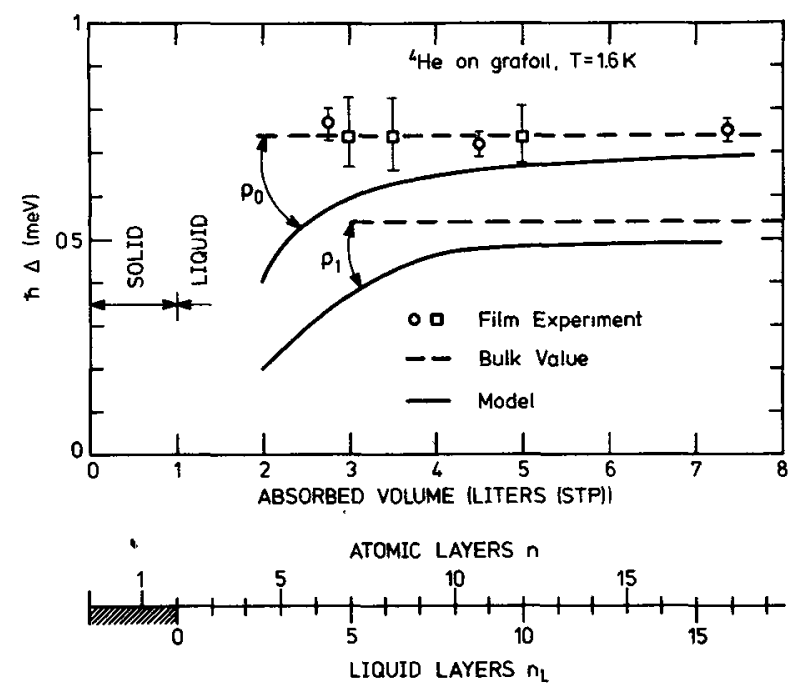

FIG. 7. - Roton energy in thin films of ${ }^{4} \mathrm{He}$, adsorbed on grafoil. The bulk value and the model (described in the text) is calculated for the two densities corresponding to saturated vapour pressure $\left(\rho_{0}\right)$ and the bulk solidification pressure of $24 \mathrm{~atm} .\left(\rho_{1}\right)$. 
transformed to an equivalent $\Delta$, shown in figure 7 for the two extreme densities $\rho=\rho_{0}$ (corresponding to saturated vapour pressure), and $\rho=\rho_{1}$ (solidification pressure). The discrepancy between the model and the experiments appears to be outside experimental uncertainty.

Hence the study of ${ }^{4} \mathrm{He}$ on grafoil reinforces the problem of relating the macroscopic properties of the superfluid to the microscopic excitations. The spacial arrangement, where several layers are necessary to give superflow, makes the neutron scattering of little relevance in the context of ideal $(2-D)$ theories, but our results do indicate that the rotons occur in a well defined portion of the film.

Acknowledgments. - The inspiring collaboration with W.D. Ellenson, J.K. Kjems, J.P. McTague, L. Passell, and $\mathrm{H}$. Taub is gratefully acknowledged.

\section{References}

[1] Taub, H., Passell, L., Kuems, J.K., Carneiro, K., MCTAgue, J.P. and DASH, J.G., Phys. Rev. Lett. 34 (1975) 654.

[2] Taub, H., Carneiro, K., KJems, J.K., Passell, L. and MCTAGUe, J.P., to be published in Phys. Rev. B.

[3] Carnetro, K., Ellenson, W.D., Passell, L., Mctague, J.P., TAUB, H., Phys. Rev. Lett. 37 (1976) 1695.

[4] Sears, V.F., Can. J. Phys. 44 (1966) 1279, 1299.

[5] KJEMS, J.K., Passell, L., Taub, H., Dash, J.G. and Novaco, A.D., Phys. Rev. B 13 (1976) 1466

[6] Warren, B.E., Phys. Rev. 59 (1941) 693.

[7] NiElsen, M., MCTAGUE, J.P. and Ellenson, W., J. Physique Colloq. 38 (1977) C4-10.

[8] Marti, C., Thorel, P., J. Physique Colloq. 38 (1977).

[9] Stirling, W.G., Scherm, R., Hilton, P.A., Cowley, R.A., J. Phys. C : Solid State Physics 9 (1976) 1643.

[10] Coulomb, J.P., BIENFAIT, M. and THOREL, P., J. Physique Colloq. 38 (1977) C4-31. MARLOW, I., THOMAS, R.K.,
Trewern, T.D. and White, J.W., J. Physique Colloq. 38 (1977) C4-19.

[11] Nielsen, M., Ellenson, W.D., in Proceedings of the Fourteenth International Conference on Low Temperature Physics, Otaniemi, Finland, 1975 (Krusius, M. and Vuorio, M., editors, North Holland), 1975, p. 437.

[12] CotTerill, R.M.J. and Pedersen, L.B., Solid State Commun. 10 (1972) 439

[13] CotTerill, R.M.J., Philos. Mag. 32 (1975) 1283.

[14] Novaco, A.D. and MCTague, J.P., J. Physique Colloq. 38 (1977) C4-116.

[15] Nicklow, R., Wakabayashi, N. and SMITh, H.G., Phys. Rev. B 5 (1972) 4951.

[16] Scholtz, J.H., MCLeAn, E.D., Rudnick, I., Phys. Rev. Lett. 32 (1974) 147.

[17] Clow, J.R. and REPPY, J.D., Phys. Rev. Lett. 16 (1966) 887.

[18] Dietrich, O.W., Graf, E.H., Huang, C.H., Passell, L., Phys. Rev. A 5 (1972) 1377.

\section{DISCUSSION}

M. SCHICK. - In view of the theorems concerning the lack of long range order at finite temperatures, what do you mean by ideal 2-D solid ?

K. CARNEIRO. - When I say ideal, I strictly speaking mean an infinite and triangular crystal. This was assumed in the $T=0$ phonon-calculation. The success of this model as compared to our experiments tells that crystals of the size of $100 \AA$ are big enough to sustain good phonons. But $100 \AA$ is much too small to illuminate the problems of real infinite crystals.
S. C. YING. - Theoretically, the mean square displacement of an infinite two dimensional solid should diverge at $T \neq 0$. How is the theoretical temperature $(\sim 55 \mathrm{~K})$ for the apparent melting transition arrived at?

K. CARNEIRO. - The computer-simulation was done on a square-array with periodic boundaries. $T$ was derived from the kinetic energy, assuming equipartition. 
A. D. Novaco. - The divergence of the rms displacement is very weak. It has been shown by W. A. Hoover that a 2-D crystal the size of the known universe has a rms divergence of $10 \AA$. Therefore, for practical experimental systems, the rms divergence can be ignored or treated with a simple cut-off procedure.

L. DOBRZYNSKI. - In answer to Pr Ying's question, it was shown (L. Dobrzynski and $I$. Lajzerowicz, Phys. Rev. B 12 (1975) 1358) that the mean square displacements of adsorbed atoms go like

$$
\left\langle u^{2}\right\rangle \propto \ln \frac{\beta^{\prime \prime}}{\beta^{\prime}}
$$

where $\beta^{\prime \prime}$ are the adsorbate-adsorbate interactions and $\beta^{\prime}$ the adsorbate-substrate interactions. So, even for $\beta^{\prime} \ll \beta^{\prime \prime}$, the $\left\langle u^{2}\right\rangle$ do not diverge.

J. A. VENABLES. - I am concerned whether your $100 \AA$ crystal size is really determined by the grafoil. Could it be determined by the defect structure of the adsorbed film. For example, this might account for the Debye-Waller anomalies and the epitaxial structure, as discussed in our paper.

K. CARneiro. - With the occurrence of new substrates has fairly conclusive shown that $L \sim 100 \AA$ is limited by the particular substrate. $N_{2}$, Ar, $H_{2}$ and $D_{2}$ on grafoil GTA all give $L=100 \pm 10$, whereas $D_{2}$ on «UCAR/ZYX» yields $L \geqslant 200 \AA$.

B. MUTAFTSCHIEV. - Are you sure that between 4.7 and $78 \mathrm{~K}$ the adsorbed matter is strictly limited to one monolayer and that the broadening of the (10) peak (Fig. 1) is not due to mobility through vacancy formation, i.e. to thermal roughness?

K. CARneiro. - No. The isotherms show that there is promotion of the second layer. But coherent neutron scattering is not directly sensitive to mobility; and our parameterization cannot distinguish between different lattice defects.

M. BIENFAIT. - You have observed a variation of the diameter of the $2 \mathrm{D}$ argon crystals as a function of $T$. Did you anneal the film before starting the observation?

K. CARnEIRO. - Yes, and the low temperature results proved to be quite dependent upon proper annealing.

R. K. THOMAS. - a) Have you measured diffraction patterns from the Ar-grafoil system at coverages greater than one ?

b) Are these patterns sufficiently different that you can readily distinguish a true monolayer from islands of, say, bi- or tri-layers ?
K. CARnEIRO. - We did measurements on larger coverages, and as far as we could tell the second and third layers were covering the whole surface. If clusters of 3-dimensional crystals we formed, the diffraction pattern would lose the characteristic trailing edge as well as the anisotropy with respect to the scattering geometry.

J. P. McTague. - (Comment on question by $R$. Thomas concerning neutron evidence on whether films form uniformly or cluster into multilayer regions.)

In the case of $\mathrm{N}_{2}$, which forms a registered $\sqrt{3} \times \sqrt{3}$ lattice for submonolayer structures, there is direct evidence from the intensity of the (10) Bragg peak as a function of filling that a uniform monolayer is formed, up to the coverage corresponding to one $\mathrm{N}_{2}$ molecule per 6 surface carbon atoms. Above that filling, the decrease in the registered (10) peak intensity indicates that the molecules are being squeezed out of this structure.

In the case of argon, which forms an incommensurate structure, the inelastic scattering pattern for submonolayer fillings is quite anisotropic: for scattering parallel to the grafoil planes, a characteristic 2-D inelastic spectrum is observed, while for perpendicular scattering only a single resonant peak is seen. However, for fillings greater than two monolayers the spectra become isotropic.

W. STEELE. -- If one naively thinks of grafoil as a collection of particles of diameter roughly equal to thickness, one would conclude that a large fraction of the exposed area would not be basal plane. In that case, it is surprising that models based on film formation exclusively on the basal plane work so well.

K. CARNEIRO. - Electronmicrographs of grafoil (type GTA) show the existence of sheets of typical dimensions of several thousand angströms. The best number for the thickness probably comes when one divides the total volume by thickness adsorption area which gives $300 \AA$. The width of the 002 reflection gives (with large uncertainty) $100 \AA$. What limits the size of the adsorbed crystals must then be lattice imperfections on the surfaces of the sheets. This picture of the grafoil is consistent with dominant basal plane adsorption.

F. A. PUTNAM. - Can one explain the observation of bulk rotons by supposing that the helium condenses in capillary form, so that one is really looking at bulk helium?

K. CARNEIRO. - Since we do see anisotropy with respect to scattering geometry, indicating that we are not dealing with isotropy large bulk-like droplets. 